\title{
High-Frequency Acoustic Noise of Lake Baikal
}

V. M. Al̆nutdinov ${ }^{a}$, V. A. Balkanov ${ }^{a}$, I. A. Belolaptikov ${ }^{g}$, L. B. Bezrukov ${ }^{a}$, N. M. Budnev ${ }^{b}$, R. V. Vasil'ev ${ }^{g}$, R. Wischnewski ${ }^{i}$, O. N. Gaponenko ${ }^{a}$, R. Yu. Gnatovskiî ${ }^{h}$, O. A. Gress ${ }^{b}$, T. I. Gress ${ }^{b}$, O. G. Grishin ${ }^{b}$, I. A. Danil'chenko ${ }^{a}$, Zh.-A. M. Dzhilkibaev ${ }^{a}$, A. A. Doroshenko ${ }^{a}$, A. N. Dyachok ${ }^{b}$, G. V. Domogatskiir ${ }^{a}$, V. A. Zhukov ${ }^{a}$, A. M. Klabukov ${ }^{a}$, A. I. Klimov ${ }^{f}$, S. I. Klimushin ${ }^{a}$,

K. V. Konishchev ${ }^{g}$, A. A. Kochanov ${ }^{b}$, A. P. Koshechkin ${ }^{a}$, V. F. Kulepov ${ }^{d}$, L. A. Kuz'michev , $^{c}$ B. K. Lubsandorzhiev ${ }^{a}$, T. Mikolajskiï , M. B. Milenin ${ }^{d}$, R. R. Mirgazov $^{b}$, S. P. Mikheev ${ }^{a}$, É. A. Osipova ${ }^{c}$, A. I. Panfilov ${ }^{a}$, A. A. Pavlov ${ }^{b}$ G. L. Pan'kov ${ }^{b}$, L. V. Pan'kov ${ }^{b}$, E. N. Pliskovskiı̆ ${ }^{g}$, V. A. Poleshchuk ${ }^{a}$, E. G. Popova ${ }^{c}$, P. G. Pokhil ${ }^{a}$, V. V. Prosin ${ }^{c}$, M. I. Rozanov ${ }^{e}$, V. Yu. Rubtsov $b$, B. A. Tarashchanskiî́ , S. V. Fialkovskiî́, A. G. Chenskiî ${ }^{b}$, B. A. Shăbonov ${ }^{a}$, Ch. Spiering ${ }^{i}$, O. Streicher ${ }^{i}$, and I. V. Yashin ${ }^{c}$

${ }^{a}$ Institute of Nuclear Research, Russian Academy of Sciences, pr. 60-letiya Oktyabrya 7a, Moscow, 119312 Russia

${ }^{b}$ Irkutsk State University, ul. K. Marksa 1, Irkutsk, 664003 Russia

${ }^{c}$ Moscow State University, Vorob’evy gory, Moscow, 119992 Russia

${ }^{d}$ Nizhni Novgorod State Technical University, ul. Minina 24, Nizhni Novgorod, 603600 Russia

e St. Petersburg State Marine Technical University, ul. Lotsmanskaya 3, St. Petersburg, 190008 Russia

${ }^{f}$ Kurchatov Institute Russian Research Center, pl. Kurchatova 1, Moscow, 123182 Russia

${ }^{g}$ Joint Institute for Nuclear Research, ul. Zholio-Kyuri 6, Dubna, Moscow oblast, 141980 Russia

${ }^{h}$ Limnological Institute, Siberian Division, Russian Academy of Sciences, ul. Ulan-Batorskaya 1, Irkutsk, 664003 Russia

${ }^{i}$ DESY-Zeuthen, Platanenallee 6, D-15738, Zeuthen, Germany e-mail: nbudnev@api.isu.ru

Received June 22, 2005

\begin{abstract}
High-frequency noise of Lake Baikal is investigated using a submersible self-contained instrument to determine the noise background for the acoustic detection of superhigh-energy neutrinos. It is found that, under stationary and uniform meteorological conditions, the integral noise power in the frequency band $1-50 \mathrm{kHz}$ is virtually independent of depth and is $10-200 \mathrm{mPa}$ or more, depending on the specific conditions. The noise itself contains multiple short pulses of different amplitudes and shapes.
\end{abstract}

PACS numbers: 43.30.Nb, 98

DOI: $10.1134 / \mathrm{S} 1063771006050010$

\section{INTRODUCTION}

The idea of acoustic detection of high-energy neutrinos dates back to the publication by G.A. Askar'yan that appeared in 1957 [1]. The potential of the method is based on the fact that the absorption of acoustic waves in water is much weaker than the absorption of Cherenkov radiation. This is especially true for fresh water, where the ultrasonic attenuation length at a frequency of about $50 \mathrm{kHz}$ exceeds $1 \mathrm{~km} \mathrm{[2].} \mathrm{The} \mathrm{second}$ fact that is favorable for the detection of acoustic signals from cascade showers at distances of hundreds of meters, or even at such long distances as several kilometers, is that the amplitude of pulses produced by showers in the near-field zone decreases only as the square root of distance, while in the far-field zone it decreases, as the reciprocal of distance from the shower
[3, 4]. Therefore, in principle, a deep-water acoustic detector of high-energy neutrinos can have a much smaller number of measuring channels compared to a Cherenkov detector with the same effective volume. The possibility of practical realization of the idea of acoustic detection depends on both the amplitude of the signal caused by the interaction of a neutrino or other superhigh-energy particle with water and the characteristics of acoustic noise in a given water basin. Actually, the very possibility (or impossibility) of acoustic detection of high-energy neutrinos and the energy detection threshold are determined by the possibility (or impossibility) of separating the signals produced by cascade showers from noise produced by other sources.

Ambient sea noise has been much investigated [5, $6]$. The most intense sources of noise are wind waves, 
(a)

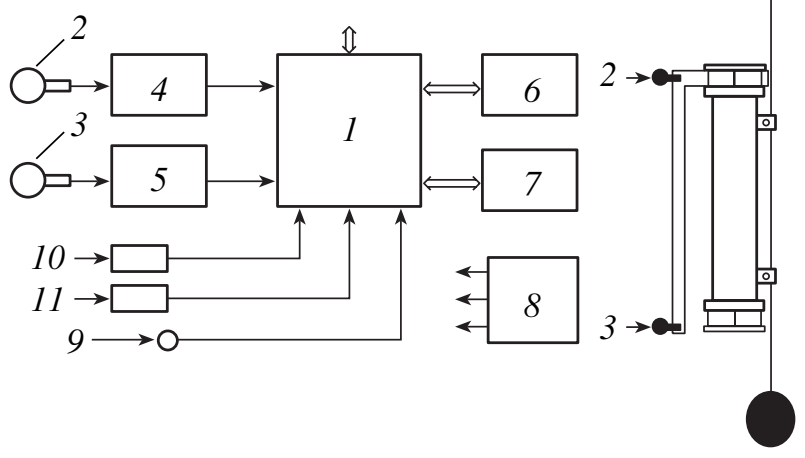

Fig. 1. Schematic representation of the self-contained instrument for acoustic noise measurements: (a) a flow chart and (b) a schematic diagram of the relative positions of two hydrophones and the module. (1) A microcontroller with a built-in A/D converter, $(2,3)$ two hydrophones, $(4,5)$ preamplifiers with low-pass filters, (6) memory, (7) clock, (8) battery power unit, $(9)$ light-emitting diode, and $(10,11)$ magnetically driven contacts for switching the instrument on and off.

wind, rain, ice, ship traffic, etc., which are located in the surface water layer; biological noise and thermal noise are generated throughout the whole water column. The bottom of a water basin can represent a source of seismic noise and noise associated with gas emission. Thermal noise represents the lower bound of underwater noise and determines the minimum threshold for acoustic neutrino detection [7]. Its spectral density at a frequency of $50 \mathrm{kHz}$ is $10^{2-1}$ and linearly grows with frequency at a rate of $6 \mathrm{~dB} /$ octave [6]. In Lake Baikal, underwater acoustic noise was quantitatively investigated in the whole range of depths only in connection with the development of the hydroacoustic coordinatemeasuring system [8] of the NT-200 Baikal deep-water neutrino telescope [10].

From the assumption that the main mechanism of acoustic signal generation by cascade showers is thermoacoustic [1], it follows that the pressure pulses produced by them should have a bipolar shape. The sign of the first half-wave of a pulse is positive if the water temperature at the site of shower development is higher than the temperature corresponding to maximum water density and the heating of water by the cascade shower energy leads to its expansion. In the opposite case, the sign of the first half-wave is negative. The expected pulse duration is on the order of several tens of microseconds and is proportional to the shower diameter. The pulse amplitude is proportional to the released energy density or inversely proportional to the heated volume at a given shower energy. Other mechanisms of acoustic signal generation by showers are also possible, such as microshock waves or bubbles [9], which may produce signals with other properties. Hence, for determining the properties of acoustic noise as the background against which cascade showers produced in water by superhigh-energy neutrinos should be detected, it is necessary to measure the amplitude-time characteristics of this noise in the high-frequency spectral region.

\section{INSTRUMENTATION}

The instrument developed for high-frequency noise measurements is schematically represented in Fig. 1. The measuring system of instrument $l$ is made on the basis of a flash microcontroller, which contains a 12-bit $\mathrm{A} / \mathrm{D}$ converter with a digitization period of $8.5 \mu$ s and a multichannel analog multiplexer. Spherical piezoceramic hydrophones 2 and 3 with a diameter of $50 \mathrm{~mm}$ have a sensitivity of $200 \mu \mathrm{V} / \mathrm{Pa}$. Preamplifiers 4 and 5 ensure a gain of $80 \mathrm{~dB}$. To suppress the low-frequency underwater acoustic noise, the amplifiers have a frequency correction with an attenuation of about $-20 \mathrm{~dB}$ per octave in the frequency band below $1 \mathrm{kHz}$. To restrict the high-frequency noise spectrum, the preamplifier outputs are connected to discrete low-pass filters with a cut-off frequency of $50 \mathrm{kHz}$. Internal memory 6 is used to store the data. Clock 7 serves for the time referencing of data. Preliminary adjustment of the mode of operation of the measuring system is performed by a PC via a series interface (RS-232C) using a loading program, which sets the length of data record and pause time intervals. The intervals were determined so as to have sufficient memory capacity for the time of submersion.

The electronic part of the instrument is enclosed in a cylindrical casing $170 \mathrm{~mm}$ in diameter, which is intended for deep-water operation. Three sealed joints are mounted on the upper cover of the instrument. Two of them serve for connecting the hydrophones, and the third has a built-in light-emitting diode 9 for controlling the performance of the measuring system. In addition, two magnetically driven contacts, 10 and 11 , are positioned under the cover, which serve to start or stop the operation of the measuring system using a small external magnet.

The performance of the instrument was checked by analyzing the results of measuring the amplitude-frequency characteristics of the channels and estimating the power spectral density of intrinsic noise. In 2003, the intrinsic noise spectrum of the instrument had several narrow peaks caused by the hard disk storage. The integral level of intrinsic noise, which was measured by connecting 30-nF capacitors instead of hydrophones to the channel inputs and then reduced to the input and expressed in pressure units, was $12.5 \mathrm{mPa}$ in the $50-\mathrm{kHz}$ frequency band in 2003 and $1.3 \mathrm{mPa}$ in the $30-\mathrm{kHz}$ band in 2004.

\section{MEASURING TECHNIQUE AND BASIC RESULTS}

In this paper, we present the results of high-frequency noise measurements which were carried out by lowering the instrument into water (i) from the ice cover during winter expeditions in March-April of 2003 and 2004 in the Southern Baikal region where the 


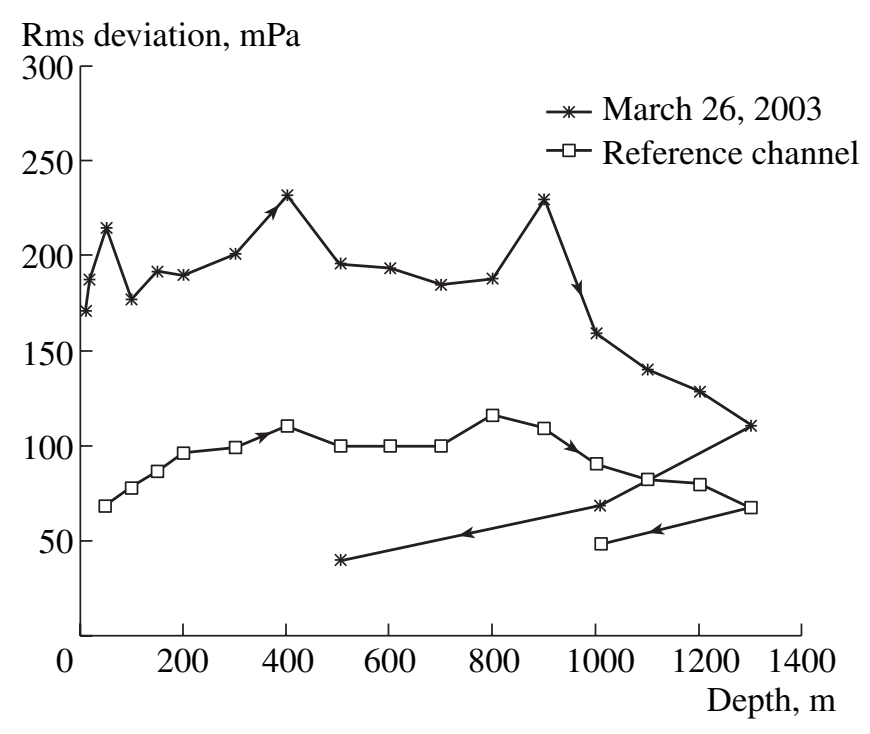

Fig. 2. Depth dependence of the integral acoustic pressure in the frequency band $1-50 \mathrm{kHz}$. The measurement of March 26, 2003, at the ice station. The lower curve represents the reference hydrophone data in relative units.

NT-200 Baikal neutrino telescope was positioned [8], (ii) from the board of the G.Yu. Vereshchagin research vessel in June of 2003 in Southern Baikal, and (iii) from the board of a boat of the Admiralteets class in August of 2004 near the NT-200 site. The characteristic depth of the lake in the regions of measurements was about 1350-1400 $\mathrm{m}$. The bottom was covered with a thick sediment layer of mainly organic origin.

In winter 2003, three cycles of measurements were carried out. The instrument was lowered from the ice into the water at a step of 20 to $200 \mathrm{~m}$ in depth and stayed for 5-10 min at each of the measuring horizons. The first measurement cycle was performed on March 26,2003 , for depths down to $1300 \mathrm{~m}$, at the ice station site, from $12: 45$ to $17: 11$ local time under sunny conditions. Digitization and recording of data were performed at a rate of $117647 \mathrm{~Hz}$ per channel within 0.28-s time intervals with a period of $10.63 \mathrm{~s}$. The distance between the hydrophones was $143 \mathrm{~cm}$. The measurements were performed at 19 horizons (on both lowering and lifting the instrument), on the average, for $5 \mathrm{~min}$. The second measurement cycle was carried out on the night of March 31 to April 1, 2003, at the same place, from 22:21 to 02:28. The measurements were performed at 16 different depths with an average measurement time of $10 \mathrm{~min}$ at each of them. The third cycle was performed on April 5, 2003, at a distance of about $600 \mathrm{~m}$ from the ice station in the daytime, from 15:00 to $17: 55$. The measurements were performed at 10 different depths for approximately $10 \mathrm{~min}$ at each of them. The noise measurements that were carried out with the research vessel in 2003 followed a similar pattern, but the duration of measurements at each individual depth was considerably smaller.
Rms deviation, $\mathrm{mPa}$

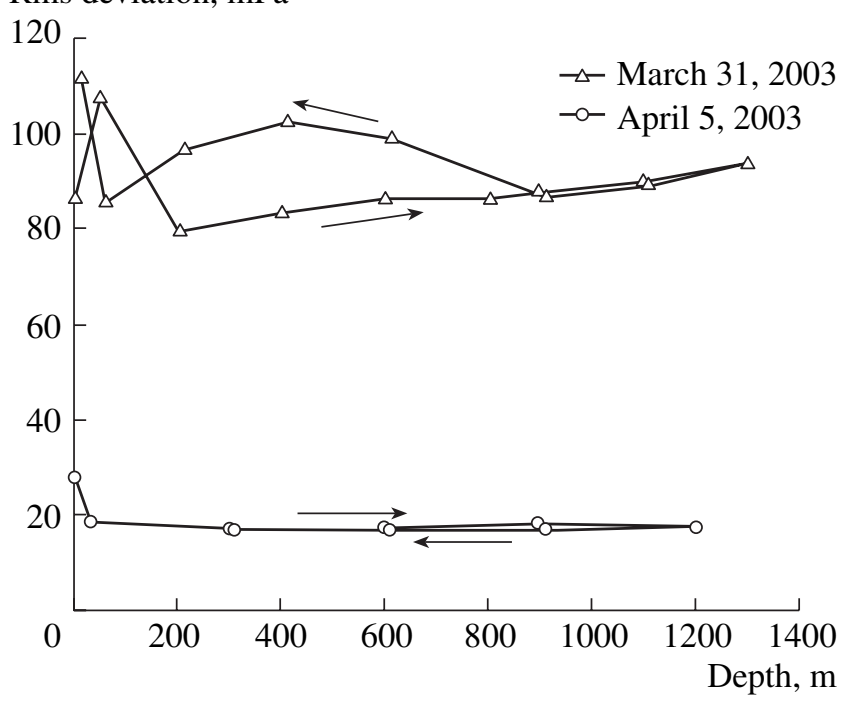

Fig. 3. Depth dependence of the integral acoustic pressure in the frequency band $1-50 \mathrm{kHz}$. The measurements of March 31, 2003, at the ice station and of April 5, 2003, at a distance of $600 \mathrm{~m}$ from the ice station.

In the ice-cover-based measurements of 2003, the noise environment was permanently monitored at a depth of $5 \mathrm{~m}$ by an additional hydrophone with another measuring system. Since the characteristics of this channel were different, data taken from the additional hydrophone did not coincide in magnitude with the data obtained by the deep-water instrument. However, they adequately represented the variations in ambient noise and also served for selecting the artefacts associated with the operation of machines at the ice station.

In 2004, another mode of measurements was used. Ice-cover-based measurements were carried out at three points lying $600 \mathrm{~m}$ away from the ice station. The instrument was lowered into the water to a fixed depth (from 50 to $1350 \mathrm{~m}$ ) and stayed there for one to three days. In August, noise measurements were performed from the board of a small boat with all of its mechanisms being turned off to provide for total silence. To avoid any influence of the boat on the measurement results, the cable that held the instrument at a depth of $250 \mathrm{~m}$ was fastened to a small buoy floating at a depth of about $1 \mathrm{~m}$ while the cable part between the boat and the buoy was loosened.

Figures 2 and 3 show the dependences of the integral noise level on the submersion depth for three cycles of measurements with lowering the instrument from the ice in 2003. In Fig. 2, the upper curve refers to the effective values of the integral pressure level fluctuations in millipascals for one of the measuring channels (for the other channel, the data are similar). The lower curve represents the variations of the signal level obtained from the reference hydrophone and reflects the time dependence of near-surface noise (in relative units) at the corresponding instants of time. Results of 


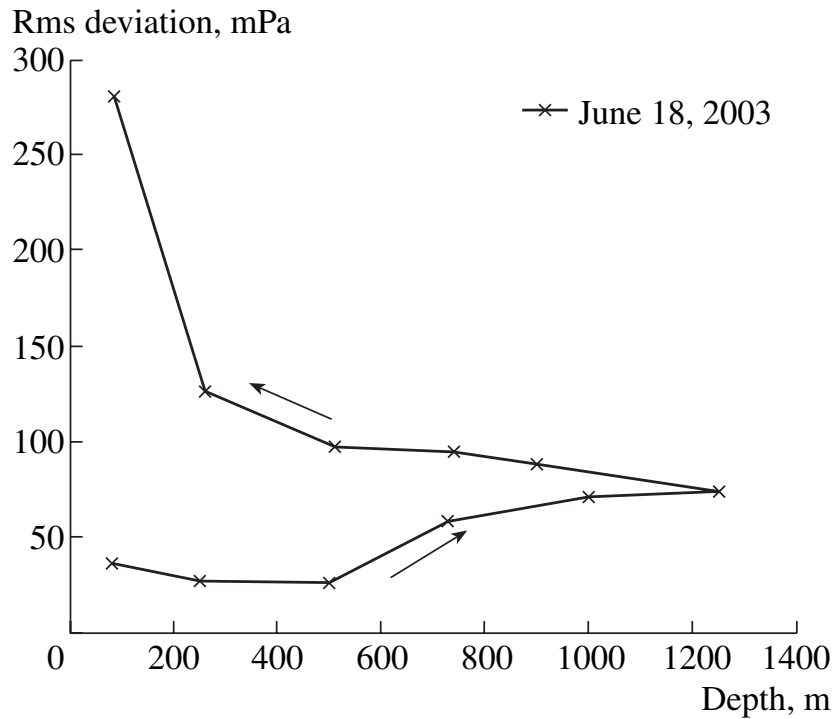

Fig. 4. Depth dependence of the integral acoustic pressure in the frequency band $1-50 \mathrm{kHz}$. The measurement of June 18 , 2003, in the southern part of Lake Baikal.

one of the noise measurements performed in summer, when the water surface is free of ice, are shown in Fig. 4.

Let us analyze these figures in more detail. The measurements that gave the results shown in Fig. 2 were started at $12: 45$, i.e., at the time of most intense heating of the ice by the sun, which caused multiple cracking of the ice. At the end of the measurement cycle, the sun was lower and the heating of the ice, as well as the noise produced by its cracking, were reduced. Presumably, this explains the fivefold decrease in the noise level detected at a depth of $500 \mathrm{~m}$ in the upward run of measurements, as compared to the results obtained in the downward run. The second submersion began at 22:28 local time and lasted for four hours. The noise level (Fig. 3) exhibited much smaller variations and was on the average 2-3 times lower than the levels detected at noon (compare with Fig. 2); however, it was still higher than the minimum level detected in the preceding cycle of measurements. An increase in the noise level at a depth of $400 \mathrm{~m}$ (within the time interval from 01:36 to 01:47) coincides with the increase in the noise level detected by the reference hydrophone at this instant of time. The third cycle of measurements (Fig. 3) was carried out under the most favorable meteorological conditions. Dense clouds prevented the heating of the air and ice by the sun, and no wind was present. In this cycle, the integral level of acoustic noise in the frequency band $1-50 \mathrm{kHz}$ did not exceed $20 \mathrm{mPa}$ and was close to the intrinsic noise level of the instrument in order of magnitude. Although in the first and second cycles, the integral noise level varied considerably, such variations presumably were related not to the variation of the measurement depth but to the intensity variations of the noise sources depending on meteorological conditions. This conclusion is suggested by com- parison with the data obtained from the reference hydrophone (Fig. 2). In the third cycle, some noise increase was observed at large depths while, near the surface, noise was found to decrease. A possible reason for this effect is considered in the following section.

Figure 4 presents the results of noise measurements on June 18, 2003, from 05:31 to 06:55. At the beginning, the noise level was close to the minimum one observed in winter (Fig. 3). A slight initial decrease in noise with lowering down to $500 \mathrm{~m}$ may possibly be related to the increase in the distance to the research vessel used for the measurements. Rain, which began at the time of the submersion to the next horizon, can explain the increase in the noise level observed in the second part of the measurements. As the surface was approached, the influence of noisy activity on board the vessel began to manifest itself.

The effect of meteorological conditions most clearly manifests itself in long-term noise measurements. In winter, as a rule, one or two noise intensity maxima are observed within $24 \mathrm{~h}$ : one in the afternoon, and one at night, when the ice temperature varies most rapidly (Fig. 5).

\section{SPECTRAL CHARACTERISTICS OF ACOUSTIC NOISE}

Figure 6 shows examples of the power spectral density (PSD) of noise. All the spectra obtained from our measurements can be classed into two groups. The first group includes the spectra with PSD of noise that decreases more or less uniformly with increasing frequency in the frequency band under study $(1-50 \mathrm{kHz})$. An example of such a spectrum, which was obtained on March 26, 2003, at a depth of $1200 \mathrm{~m}$, is shown in Fig. 6a (the growth near $30 \mathrm{kHz}$ is associated with the manifestation of the resonance properties of the hydrophone). This kind of noise produced by multiple sources uniformly distributed near the surface was observed under stable and calm meteorological conditions. The qualitative behavior of the PSD of noise does not contradict the empirical frequency dependence typical of the ocean [12]. However, the slope of the spectra is only about 5-6 dB/octave on the average. In winter, the spectra are usually somewhat steeper than in summer. Figure 7 shows the slopes of the PSD of noise obtained from 34 measurements. The "whiskers" in the plot do not reflect the error in the determination of the slope of the spectrum, but rather the variation of the slope in calculations from the data for different time intervals within one submersion. As a rule, the whiskers are smaller for measurements of smaller duration. Hence, they are greater for the measurements performed under the ice cover in 2004 (submersions 1935 ), which lasted for several days rather than hours, as in 2003 (submersions 1-3). In some of the submersions, such as 3 and 9 (their spectra are shown in Figs. 3 and 4, respectively), the increase in the size of whiskers is associated with the nonmonotonicity of the spectra. 


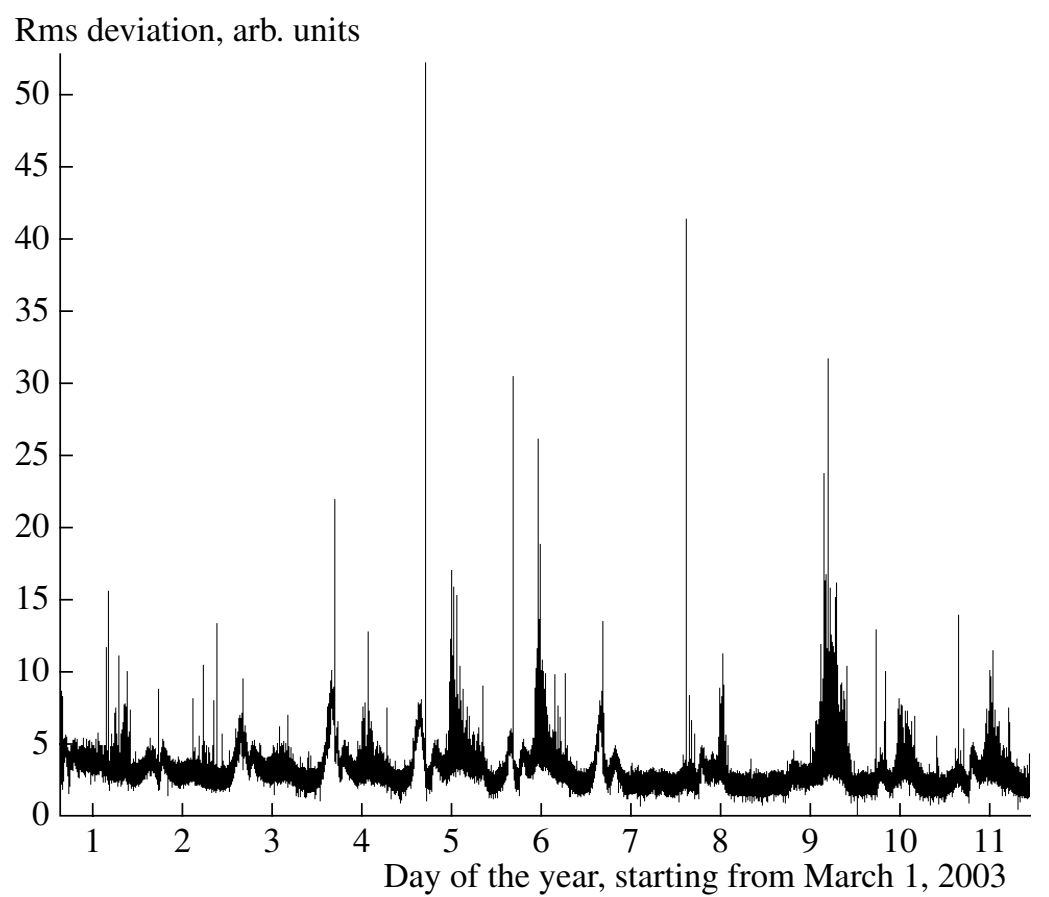

Fig. 5. Variations of the integral acoustic pressure level in early March of 2003 at a depth of $1000 \mathrm{~m}$ (in relative units).

Such spectra, which contain some specific features in certain frequency bands, belong to the second group. These spectra are observed under nonstationary and nonuniform meteorological conditions. Spectral features may also arise in the presence of some noise sources with specific spectra not far from the measurement site. For example, the spectrum shown in Fig. 6a (measurement of April 5, 2003) exhibits a considerable noise level increase in the region of several kilohertz. Possibly, this feature is related to the noise produced by the gas emission from bottom sediments, which often occurs in Baikal [11]. Indeed, researchers from the Limnological Institute (Siberian Division, Russian Academy of Sciences) did reveal an elevated methane content in water in this part of the lake; moreover, similar spectra were observed near the points of gas emission in other regions. Figure $6 \mathrm{~b}$ clearly shows the change in the PSD of noise with an increase in the intensity of rain. As a rule, the spectra of signals received from the two hydrophones agree well with each other. However, when the measurements were carried out within several meters of the surface, a considerable difference was observed between the spectra received from the upper and lower hydrophones. This testifies to a strong vertical spatial inhomogeneity of the acoustic noise field near the ice cover (the effect of the underice sound channel).

We also note that, in the measurements performed under stable meteorological conditions and in the absence of quasi-local noise sources (the spectra of the first group), we do not observe a relative decrease in the high-frequency spectral components with increasing depth, which should occur in salt water. The latter fact is explained by the weaker attenuation of high-frequency waves in fresh water. Figure 8 shows smoothed dependences of the acoustic noise amplitude for several depths in the frequency band $22.22-44.44 \mathrm{kHz}$ for submersions from the ice cover during the expedition of 2004.

\section{PULSED NOISE CHARACTERISTICS}

The background for acoustic detection of highenergy showers is primarily represented by pulsed noise with small pulse duration. Figure 9 shows the results of the statistical analysis of data obtained from the measurements performed at a depth of $250 \mathrm{~m}$ under calm weather conditions in August of 2004, i.e., under the most favorable conditions for detecting weak signals. The first step of this analysis was the elimination of the data that contained high-amplitude noise falling beyond the limits of the dynamic range of the A/D converter. Then, we eliminated electronic stray signals and hardware errors manifesting themselves as overshoots in both channels simultaneously or with a delay equal to one time-quantization step. Figure 9a shows the distribution of short (with durations of several tens of microseconds) acoustic pulses in the number of change of sign of the acoustic pressure amplitude when passing through zero. From this histogram, it follows that unipolar pressure jumps predominate. The number of bipolar pulses, which is the type of pulses that can be generated by cascade showers, is an order of magnitude smaller than the number of unipolar ones. Figure $9 b$ 

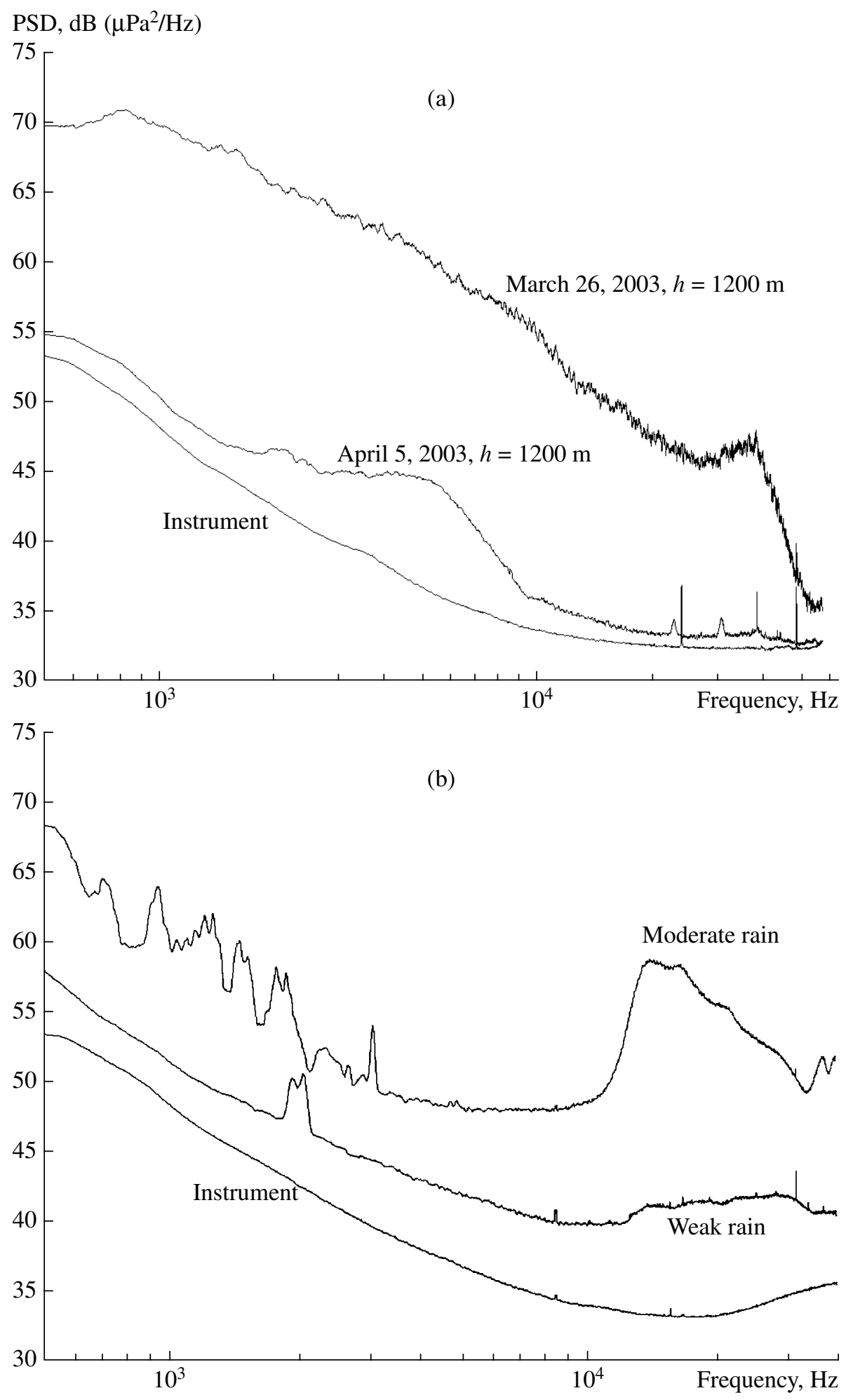

Fig. 6. Power spectral density of noise: (a) measurements of March 26, 2003, and April 5, 2003, at a depth of $1200 \mathrm{~m}$; (b) measurement of June 18, 2003, at a depth of $500 \mathrm{~m}$ (the lower curve refers to the downward run, and the upper curve, to the upward run).

shows the distribution of bipolar pulses in amplitude in units of rms deviations, and Fig. 9c shows their distribution in duration.
Thus, the search for acoustic pulses produced by super-high energy cascade showers occurs against a considerable background, which substantially increases the 


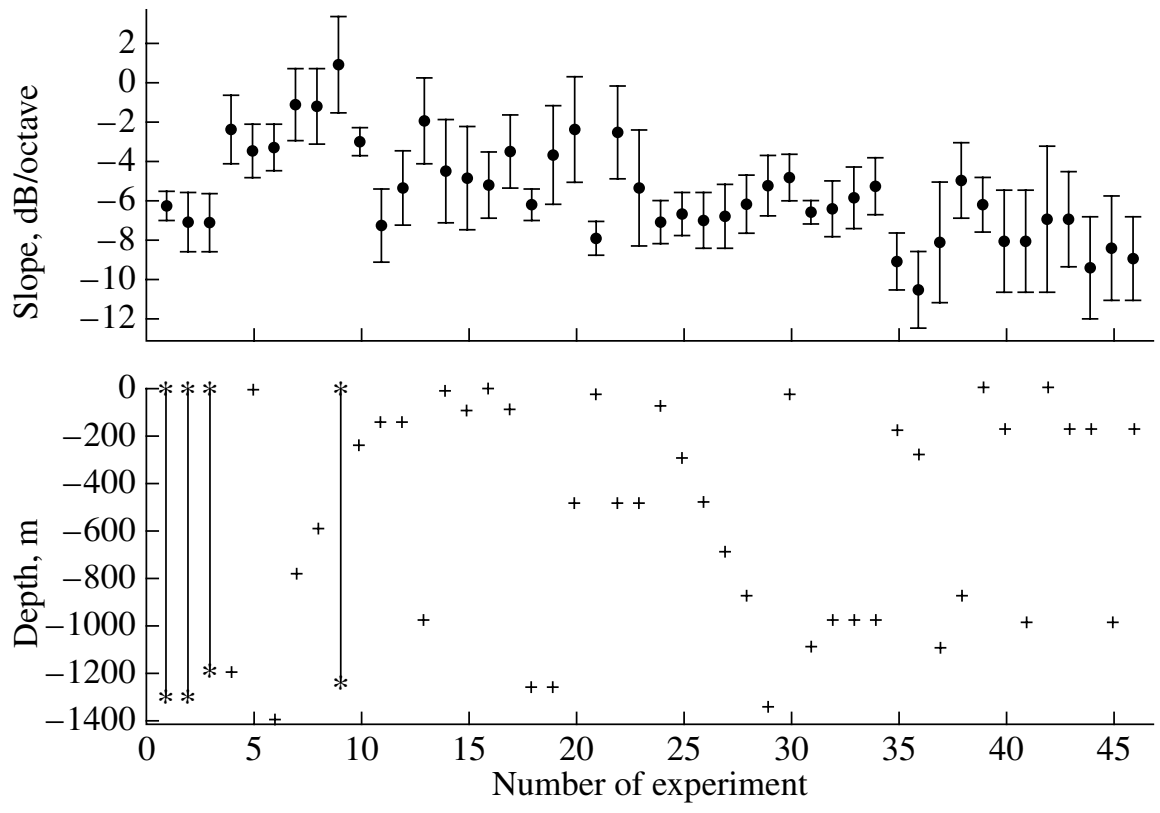

Fig. 7. Slope of the power spectral density of high-frequency noise at the depths specified in the lower plot. Experiments 4-17 were performed in summer, and all the other experiments, in the presence of ice cover.

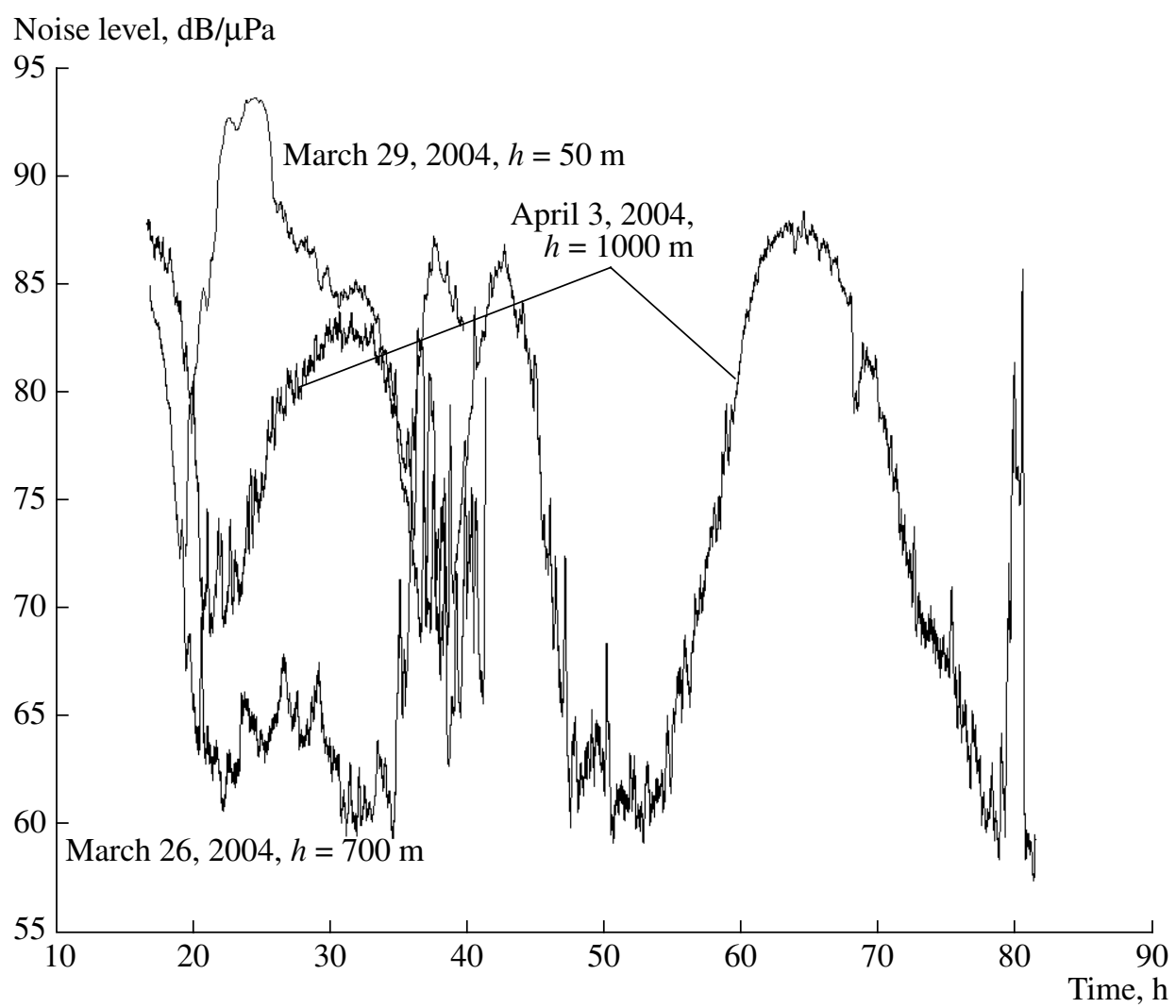

Fig. 8. Smoothed time dependences of acoustic noise amplitude for several depths in the frequency band $22.22-44.44 \mathrm{kHz}$.

detection threshold. A similar problem arose in the SAUND experiment [13], where an array of 7 hydrophones spaced at approximately $1 \mathrm{~km}$ and positioned at a depth of about 1600 m near the Bahama Islands was used to detect acoustic signals from neutrinos. In our instrument, the distance between the hydrophones is 

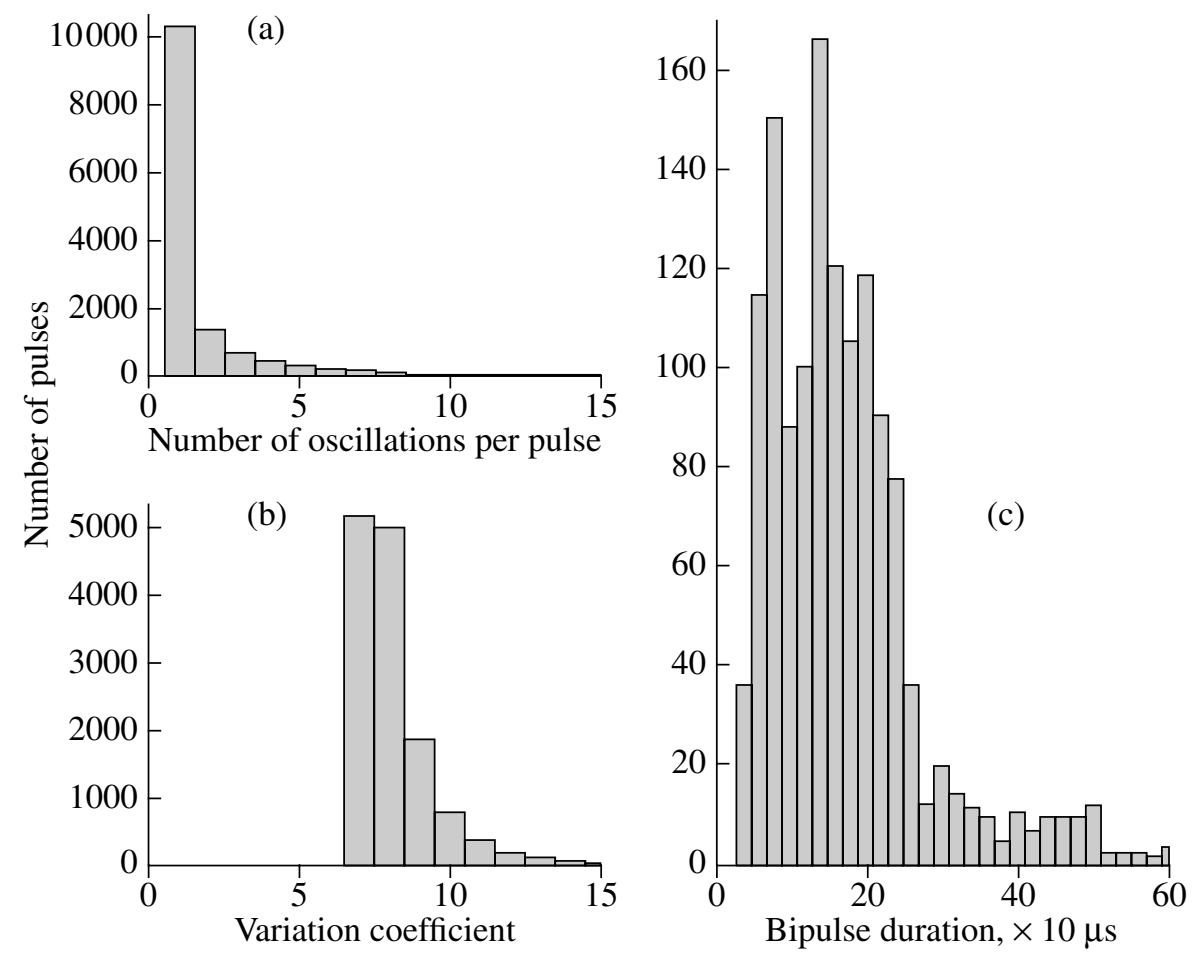

Fig. 9. Statistical characteristics of short acoustic pulses: (a) distribution in the number of changes of sign of the acoustic pressure amplitude, (b) distribution in amplitude (in rms deviation units), and (c) distribution in duration for bipolar pulses.
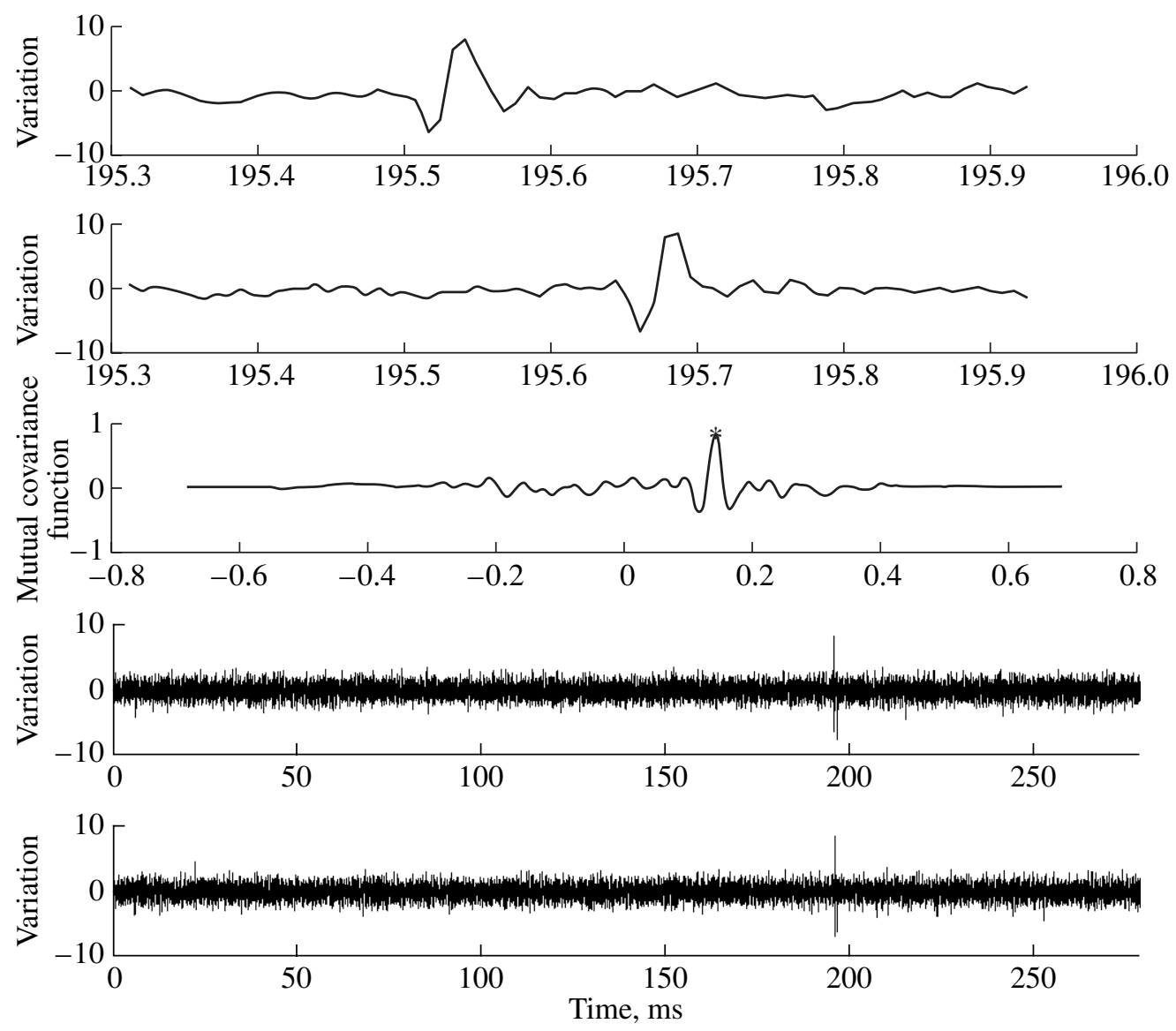

Fig. 10. Bipolar pulse recorded on April 1, 2003, at a depth of $600 \mathrm{~m}$. 
about $1 \mathrm{~m}$. This allows an efficient selection of data using the correlation analysis. The latter showed that the overwhelming majority of acoustic pressure bursts manifesting themselves as short bipolar pulses cannot be interpreted as signals from remote quasi-local sources but result from the interference of acoustic waves. Nevertheless, we found several cases where the signals from two hydrophones were strongly correlated, had amplitudes exceeding three rms deviations of noise for a given fragment of data, and had a shape close to that expected for signals from cascade showers.

A corresponding example is shown in Fig. 10. Here, the two lower plots represent a noise realization record in the two channels of the instrument. The two upper plots show fragments of the lower plots that were chosen by the selector program and stretched along the abscissa axis. The abscissa axis represents the numbers of readings in the realization (the sampling interval is $8.5 \mu \mathrm{s}$ ), and the ordinate axis, the relative amplitude in rms deviation units. The middle plot shows the mutual covariance function for the upper fragments. Here, the scale factor along the abscissa axis is 0.05 of the time sampling interval, because we used spline interpolation to reduce the error in the relative signal delay. The measurements were performed on April 1, 2003, with the instrument lowered from the ice cover to a depth of $600 \mathrm{~m}$. The presence of two recording channels spaced in depth allowed us to estimate the zenith angle of the wave vector direction for a given pulse, which proved to be $77.7^{\circ}$. The actual direction to the source of sound depends on the distance to it and the refraction, which is governed by the sound velocity profile [8].

\section{CONCLUSIONS}

Thus, the results of studying high-frequency noise of Lake Baikal testify to a rather complicated structure of the noise field and to the presence of a wide variety of sound sources. A number of the general properties of noise, such as the weak depth dependence and the slope of the power spectral density of noise, are similar to the corresponding characteristics of ambient noise in the ocean. According to the results of our measurements, the most intense noise source in Baikal is the ice cover, or, more precisely, the cracking of ice that occurs under the effect of temperature variation and other factors. In winter, when the lake is covered with ice, the mean sound pressure level in the frequency band $1-50 \mathrm{kHz}$ is $0.1-0.2 \mathrm{~Pa}$, which is several times higher than the corresponding value observed in summer, when the surface of the lake is free of ice. Moreover, only a few measurements performed in the absence of ice showed integral noise levels higher than $0.1 \mathrm{~Pa}$, whereas, in winter, the noise levels reached $1 \mathrm{~Pa}$. In winter, as a rule, the noise level exhibits considerable diurnal variations; on quiet cloudy days, the minimum noise level is equal to or even lower than the summer minimum and does not exceed $10 \mathrm{mPa}$. The integral level of natural noise in Baikal weakly depends on depth.
Under stable conditions, at all of the depths, the power spectral density of noise almost uniformly decreases by $5-6 \mathrm{~dB}$ as the frequency increases by one octave in the frequency band $1-50 \mathrm{kHz}$. During a storm, or rain, or intense motion of ice, the noise spectrum becomes nonstationary and nonuniform; sometimes, it exhibits more or less wide peaks in certain frequency bands.

The time-pulse structure of the noise is rather complicated. A great number of short (with durations of 10 $\mu$ s and over) pulses is observed, including bipolar ones, which represent a substantial background for the acoustic detection of neutrinos. Correlation analysis showed that most of these short pulses result from the interference of acoustic waves. However, it is also possible that some of the detected short pulses were generated by quasi-local sound sources.

At the next stage of studying the properties and nature of Baikal noise, in particular, with a view to acoustic detection of superhigh-energy neutrinos, we plan to use a four-channel instrument whose hydrophones will be positioned at the vertices of a regular pyramid with $1 \mathrm{~m}$ sides. In this instrument, the sampling rate will be two times higher to provide for a more accurate measurement of the short pulse characteristics. The instrument will be mounted on one of the floating buoy stations belonging to the deep-water NT-200+ Baikal neutrino telescope system to carry out long-term measurements, including those with the use of a control signal from NT-200+, which will be generated at the instants of detecting intense bursts of Cherenkov radiation. The data communication with the shore station will be realized through a cable using megabit modems. We expect that four-hydrophone measurements will allow us to suppress to a considerable extent the effect of interference and other chance factors on the detection of acoustic signals from quasi-local sources located in the water column.

\section{ACKNOWLEDGMENTS}

We are grateful to the board of directors of the Limnological Institute, Siberian Division, Russian Academy of Sciences, for allowing us to perform the measurements on board of the G.Yu. Vereshchagin research vessel. This work was supported by the Federal Agency of Science and Innovations of Russian Federation, the Federal Agency of Education of Russian Federation, the Ministry of Science and Education of Germany, the Russian Foundation for Basic Research (project nos. 02-02-17427, 03-02-310011, 04-02-31003, 04-02-31006, and 05-02-16593), the Russian Presidential Grant no. NSh-1828.2003.2, the program "Development of the Scientific Potential of Higher Educational Institutions" (project no. 4006), the INTAS grant no. 01-23-09, and the Department of Water Resources of UNESCO. 


\section{REFERENCES}

1. G. A. Askar'yan, At. Energ. 3 (8), 152 (1957).

2. C. S. Clay and H. Medwin, Acoustical Oceanography (Wiley, New York, 1977; Mir, Moscow, 1980).

3. G. A. Askariyan, V. A. Dolgoshein, A. N. Kalinovsky, and M. V. Mokhov, Nucl. Instrum. Methods 164 (2), 267 (1979).

4. J. G. Learnd, Phys. Rev. D 19, 3293 (1979).

5. V. O. Knudsen, R. S. Alford, and J. W. Emling, J. Mar. Res. 7, 410 (1948).

6. A. V. Furduev, in Ocean Acoustics (Nauka, Moscow, 1974), pp. 615-691 [in Russian].

7. R. Mellen, J. Acoust. Soc. Am. 25, 478 (1952).
8. V. M. Aĭnutdinov et al., Akust. Zh. 51, 721 (2005) [Acoust. Phys. 51, 619 (2005)].

9. L. M. Lyamshev, Usp. Fiz. Nauk 162 (4), 43 (1992) [Sov. Phys. Usp. 32, 276 (1992)].

10. I. A. Belolaptikov, L. B. Bezrukov, B. A. Borisovets, et al., Astropart. Phys. 7, 263 (1997).

11. N. G. Granin and L. Z. Granina, Geol. Geofiz. 43, 609 (2002).

12. A. V. Furduev, Vopr. Sudostr., Ser. Akust., No. 10, 45 (1973).

13. J. Vandenbroucke, G. Gatta, and N. Lihtinen, astro$\mathrm{ph} / 0406105 \mathrm{v} 1$.

Translated by E. Golyamina 